\title{
Risk of subsequent ischemic and hemorrhagic stroke in patients hospitalized for immune- mediated diseases: a nationwide follow-up study from Sweden
}

Bengt Zöller ${ }^{1 *}$, Xinjun Li ${ }^{1}$, Jan Sundquist ${ }^{1,2}$ and Kristina Sundquist ${ }^{1}$

\begin{abstract}
Background: Certain immune-mediated diseases (IMDs) have been associated with increased risk for cardiovascular disorders. The aim of the present study was to examine whether there is an association between 32 different IMDs and first hospitalization for ischemic or hemorrhagic stroke.

Methods: All individuals in Sweden hospitalized with a main diagnosis of IMD (without previous or coexisting stroke), between January 1, 1987 and December 31, $2008(n=216,291)$, were followed for first hospitalization for ischemic or hemorrhagic stroke. The reference population was the total population of Sweden. Adjusted standardized incidence ratios (SIRs) for ischemic and hemorrhagic stroke were calculated.

Results: Totally 20 and 15 of the 32 IMDs studied, respectively, were associated with an increased risk of ischemic and hemorrhagic stroke during the follow-up. The overall risks of ischemic and hemorrhagic stroke during the first year after hospitalization for IMD were 2.02 (95\% Cl 1.90-2.14) and 2.65 (95\% Cl 2.27-3.08), respectively. The overall risk of ischemic or hemorrhagic stroke decreased over time, to 1.50 (95 \% Cl 1.46-1.55) and 1.83 (95 \% Cl 1.69-1.98), respectively, after $1-5$ years, and 1.29 (95\% Cl 1.23-1.35) and 1.47 (95\% Cl 1.31-1.65), respectively, after 10+ years. The risk of hemorrhagic stroke was $\geq 2$ during the first year after hospitalization for seven IMDs: ankylosing spondylitis $(S I R=8.11)$, immune thrombocytopenic purpura ( $S I R=8.60)$, polymyalgia rheumatica $(S I R=2.06)$, psoriasis $(S I R=2.88)$, rheumatoid arthritis $(S I R=3.27)$, systemic lupus erythematosus $(S I R=8.65)$, and Wegener's granulomatosis $(S I R=5.83)$. The risk of ischemic stroke was $\geq 2$ during the first year after hospitalization for twelve IMDs: Addison's disease $(S I R=2.71)$, Crohn's disease $(S I R=2.15)$, Grave's disease $(S I R=2.15)$, Hashimoto's thyroiditis $(S I R=2.99)$, immune thrombocytopenic purpura $(S I R=2.35)$, multiple sclerosis $(S I R=3.05)$, polymyositis/ dermatomyositis $(S I R=3.46)$, rheumatic fever $(S I R=3.91)$, rheumatoid arthritis $(S I R=2.08)$, Sjögren's syndrome $(S I R=2.57)$, systemic lupus erythematosus $(S I R=2.21)$, and ulcerative colitis $(S I R=2.15)$.
\end{abstract}

Conclusions: Hospitalization for many IMDs is associated with increased risk of ischemic or hemorrhagic stroke. The findings suggest that several IMDs are linked to cerebrovascular disease.

\footnotetext{
* Correspondence: bengt.zoller@med.lu.se

'Center for Primary Health Care Research, Lund University/Region Skåne, Clinical Research Centre, Floor 11, Building 28, Entrance 72, Skåne University Hospital, 205 02, Malmö, Sweden

Full list of author information is available at the end of the article
} 


\section{Background}

Ischemic and hemorrhagic stroke are major causes of morbidity and mortality worldwide [1]. During recent years it has become clear that systemic inflammation may enhance atherogenesis [2-4]. Immune-mediated diseases (IMDs) are a heterogenous group of diseases that are characterized by acute or chronic inflammation [2-8]. Some IMDs have been associated with an increased risk for cardiovascular disease [2-8]. IMDs may increase the cardiovascular disease risk through different mechanisms such as autoreactive lymphocytes, autoantibodies, autoantigens, epigenetic mechanisms, and inflammation driving the formation, progression and rupture of atherosclerotic plaques [2-8]. Inflammation may also affect the thrombotic risk by suppressing fibrinolysis, upregulating procoagulants, and downregulating anticoagulants [7]. Thus, certain IMDs such as rheumatoid arthritis (RA) [3,5,6,8-12] and systemic lupus erythematosus (SLE) $[3,5,6,8,13-15]$ have been associated with an increased risk of cardiovascular disease. Enhanced atherogenesis has also been indicated in other IMDs such as Sjögren's disease [3,5,6,16], systemic vasculitis $[3,5]$, inflammatory bowel disease $[3,5,8,17]$, and psoriasis $[8,18]$. As a consequence of this, the risk of stroke has been reported to be increased in patients with systemic lupus erythematosus [19] and rheumatoid arthritis [20].

We hypothesized that not only IMDs such as SLE and RA, but also a number of other less well-studied IMDs have an increased risk of cardiovascular disease. More specifically, we aimed at determining whether IMDs increase the risk for hospitalized ischemic or hemorrhagic stroke. In a nationwide follow-up from 1987-2008 we have estimated the risk of hospitalization with stroke in patients hospitalized with 32 different IMDs without previous or coexisting stroke.

\section{Methods}

This study was approved by the Ethics Committee of Lund University, Sweden. Data used in this study contained information on all individuals registered as residents of Sweden [21]. It included individual-level information on age, sex, occupation, geographic region of residence, hospital diagnoses, and dates of hospital admissions in Sweden (1964-2008), as well as date of emigration, and date and cause of death [21]. The dataset was constructed using several national Swedish data registers (reviewed by Rosen and Hakulinen) [22], including, but not limited to, the Swedish National Population and Housing Census (19601990), the Total Population Register, the Multi-Generation Register, and the Swedish Hospital Discharge Register [23]. The data were released to us from the National Board of Health and Welfare and Statistics Sweden.

Information retrieved from the various registers was linked, at the individual level, via the national 10-digit personal identification number assigned to each resident of Sweden for his or her lifetime. Registration numbers were replaced by serial numbers to preserve anonymity. As well as being used to track all records in the database at the individual level, these serial numbers were used to check that individuals with hospital diagnoses of ischemic or hemorrhagic stroke appeared only once during the follow-up (for the first hospital diagnosis of ischemic or hemorrhagic stroke during the study period).

The follow-up period for analysis of data in the present study started on January 1, 1987 and continued until hospitalization for ischemic or hemorrhagic stroke, death, emigration, or the end of the study period (December 31, 2008). Data for first hospitalization for ischemic or hemorrhagic stroke during the study period were retrieved from the Hospital Discharge Register (1987-2008). This study did not include data for hospital outpatients or patients treated at primary health care centers.

\section{Predictor variable}

The predictor variable was hospitalization for an IMD, diagnosed according to ICD-7, ICD-8, ICD-9, and ICD10 (Additional file 1 Table S1).

\section{Outcome variable}

Diagnosis of ischemic stroke was based on the 9th, and 10th revisions of the International Classification of Diseases (ICD-9, and ICD-10). Cases of ischemic stroke were identified using the following ICD codes: 433, 434, 435, 437.0, and 437.1 (ICD-9); and I63 (not I636), I65, I66, I67.2, and I67.8 (ICD-10).

Diagnosis of hemorrhagic stroke was also based on ICD-9, and ICD-10. Cases of hemorrhagic stroke were identified using the following ICD codes: 431 and 432 (ICD-9); and I61 and I62 (ICD-10).

\section{Individual-level variables adjusted for in the model}

The individual-level variables were sex, age, time period, geographic region of residence, socioeconomic status (SES), and comorbidity.

Sex: male or female.

Age was divided into 5-year categories. Subjects of all ages were included in the study.

Time period was divided into five time periods in order to allow for adjustment for any change in hospitalization rates over time: 1987-1991, 1992-1996, 1997-2001, 2002-2008.

Geographic region of residence was included as an individual-level variable to adjust for possible differences in hospital admissions for ischemic or hemorrhagic stroke between different geographic regions in Sweden. It was categorized as: 1) large city (city with a population of $>200,000$ (i.e., Stockholm, Gothenburg, or Malmo); 2) Southern Sweden (both rural and urban); and 3) Northern Sweden (both rural and urban). 
Occupation was used as a proxy for SES. We classified each individual's occupation into one of six categories: 1) blue-collar worker, 2) white-collar worker, 3) professional, 4) self-employed, 5) farmer, and 6) non-employed (Individuals without paid employment). Homemakers and students without an occupation were categorized on the basis of their husband's, father's or mother's occupation. If that was not possible, they were included in the "nonemployed" category. For individuals aged $<20$ years, parental occupation was used.

Comorbidity was defined as the first hospital diagnosis at follow up (1987-2008) of the following: 1) chronic lower respiratory diseases (490-496 (ICD-9), and J40-J49 (ICD-10)); 2) obesity (278A (ICD-9), and E65-E68 (ICD10)); 3) alcoholism and alcohol-related liver disease (291 and 303 (ICD-9), and F10 and K70 (ICD-10)); 4) type 2 diabetes mellitus (250 (age $>29$ years) (ICD-9), and E11E14 (ICD-10)); 5) hypertension (401-405 (ICD-9), and I10-I15 (ICD-10)); 6) atrial fibrillation (427D (ICD-9), and I48 (ICD-10)); 7) heart failure (428 (ICD-9), and I50 (ICD10)); 8) renal disease (580-591 and 753B (ICD-9), and N00-N19, Q61 (ICD-10)); 9) sepsis (036,038 (ICD-9), and A39-A41 (ICD-10)); and 10) coronary heart disease (410414 (ICD-9), and I20-I25 (ICD-10)).

\section{Statistical analysis}

Person-years at risk (i.e., number of persons at risk multiplied by time at risk) were calculated from the time at which subjects were included in the study (in 1987 or later) until first hospitalization for ischemic or hemorrhagic stroke, death, emigration, or the end of the study period. Person years for IMD patients were calculated from discharge of first hospitalization for IMD (IMD patients with previous stroke before the first IMD hospitalization or at the same hospitalization as the first IMD hospitalization, were excluded). The expected number of cases was based on the number of cases in the reference group. SIRs were calculated as the ratio of observed (O) and expected (E) number of ischemic or hemorrhagic stroke cases using the indirect standardization method [24]:

$$
S I R=\frac{\sum_{j-1}^{J} o_{j}}{\sum_{j-1}^{J} n_{j} \lambda_{j}^{*}}=\frac{o}{E^{*}},
$$

Where $o=\sum o_{j}$ denotes the total observed number of cases in the study group; $E^{*}$ (expected number of cases) is calculated by applying stratum-specific standard incidence rates $\left(\lambda_{j}^{*}\right)$ obtained from the reference group to the stratum-specific person-years $(n)$ of risk for the study group; ${ }_{j}^{o}$ represents the observed number of cases that the cohort subjects contribute to the jth stratum; and $\mathrm{J}$ represents the strata defined by cross-classification of the following adjustment variables: age, sex, time period, SES, geographic region of residence, and comorbidity[24]. Ninety-five percent confidence intervals (95\% CIs) were calculated assuming a Poisson distribution [24]. All analyses were performed using SAS version 9.2 (SAS Institute, Cary, NC, USA).

\section{Results}

Table 1 shows the number of people admitted to hospital with each of the selected IMDs during the study

Table 1 Number of cases hospitalizations of IMD and related conditions, 1987-2008

\begin{tabular}{|c|c|c|c|c|c|c|}
\hline \multirow[b]{2}{*}{ Immune-mediated disease } & \multicolumn{2}{|c|}{ Men } & \multicolumn{2}{|c|}{ Women } & \multicolumn{2}{|l|}{ All } \\
\hline & $\mathrm{No}$ & $\%$ & No & $\%$ & No & $\%$ \\
\hline Addison disease & 862 & 1.05 & 1190 & 0.89 & 2052 & 0.95 \\
\hline Amyotrophic lateral sclerosis & 2376 & 2.89 & 2055 & 1.53 & 4431 & 2.05 \\
\hline Ankylosing spondylitis & 2416 & 2.94 & 1061 & 0.79 & 3477 & 1.61 \\
\hline $\begin{array}{l}\text { Autoimmune hemolytic } \\
\text { anemia }\end{array}$ & 312 & 0.38 & 391 & 0.29 & 703 & 0.33 \\
\hline Behcet disease & 146 & 0.18 & 138 & 0.10 & 284 & 0.13 \\
\hline Celiac disease & 2639 & 3.21 & 4249 & 3.17 & 6888 & 3.18 \\
\hline Chorea minor & 10 & 0.01 & 25 & 0.02 & 35 & 0.02 \\
\hline Crohn disease & 9522 & 11.58 & 10700 & 7.98 & 20222 & 9.35 \\
\hline Diabetes mellitus type I & 9068 & 11.02 & 7664 & 5.72 & 16732 & 7.74 \\
\hline Discoid lupus erythematosus & 54 & 0.07 & 200 & 0.15 & 254 & 0.12 \\
\hline Grave disease & 3764 & 4.58 & 18298 & 13.65 & 22062 & 10.20 \\
\hline Hashimoto thyroiditis & 1440 & 1.75 & 5115 & 3.82 & 6555 & 3.03 \\
\hline $\begin{array}{l}\text { Immune thrombocytopenic } \\
\text { purpura }\end{array}$ & 1905 & 2.32 & 2039 & 1.52 & 3944 & 1.82 \\
\hline Localized scleroderma & 90 & 0.11 & 422 & 0.31 & 512 & 0.24 \\
\hline Lupoid hepatitis & 115 & 0.14 & 274 & 0.20 & 389 & 0.18 \\
\hline Multiple sclerosis & 3492 & 4.25 & 6892 & 5.14 & 10384 & 4.80 \\
\hline Myasthenia gravis & 935 & 1.14 & 1149 & 0.86 & 2084 & 0.96 \\
\hline Pernicious anemia & 1663 & 2.02 & 1868 & 1.39 & 3531 & 1.63 \\
\hline Polyarteritis nodosa & 437 & 0.53 & 386 & 0.29 & 823 & 0.38 \\
\hline Polymyalgia rheumatica & 5313 & 6.46 & 11183 & 8.34 & 16496 & 7.63 \\
\hline Polymyositis/dermatomyositis & 404 & 0.49 & 667 & 0.50 & 1071 & 0.50 \\
\hline Primary biliary cirrhosis & 124 & 0.15 & 675 & 0.50 & 799 & 0.37 \\
\hline Psoriasis & 4471 & 5.44 & 4558 & 3.40 & 9029 & 4.17 \\
\hline Reiter disease & 280 & 0.34 & 58 & 0.04 & 338 & 0.16 \\
\hline Rheumatic fever & 236 & 0.29 & 228 & 0.17 & 464 & 0.21 \\
\hline Rheumatoid arthritis & 12080 & 14.69 & 32531 & 24.27 & 44611 & 20.63 \\
\hline Sarcoidosis & 2847 & 3.46 & 2518 & 1.88 & 5365 & 2.48 \\
\hline Sjören syndrome & 125 & 0.15 & 1175 & 0.88 & 1300 & 0.60 \\
\hline Systemic lupus erythematosus & s 742 & 0.90 & 3437 & 2.56 & 4179 & 1.93 \\
\hline Systemic sclerosis & 402 & 0.49 & 1356 & 1.01 & 1758 & 0.81 \\
\hline Ulcerative colitis & 12963 & 15.76 & 10647 & 7.94 & 23610 & 10.92 \\
\hline Wegener granulomatosis & 1025 & 1.25 & 884 & 0.66 & 1909 & 0.88 \\
\hline All & 82258 & 100.00 & 134033 & 3100.00 & 216291 & 100.00 \\
\hline
\end{tabular}


period. IMD patients with previous stroke before first hospitalization for IMD or stroke at the same time as first IMD hospitalization were excluded from Table 1. Totally 8113 IMD patient with previous or coexisting ischemic stroke and 1416 with hemorrhagic stroke were excluded. A total of 216,291 individuals were hospitalized with an IMD (82,258 males and 134,033 females) (Table 1). The three most common immune-mediated diseases were rheumatoid arthritis (44,611 cases), ulcerative colitis $(23,610)$, and Graves' disease $(22,062)$. Totally 66,509 patients with ischemic stroke and 428,031 patients with hemorrhagic strokes from 1987-2008 were included (Table 2), of whom 10,905 (9,437 ischemic and 1,468 hemorrhagic strokes) were subsequently admitted to hospital after a first hospitalization for IMD (Table 2). The comorbidities (defined as main or second hospital diagnosis) adjusted for are presented in Table 2.

\section{Hemorrhagic stroke}

A total of 66,509 individuals were hospitalized with a main diagnosis of hemorrhagic stroke (Table 2), of whom 1,468 ( $2 \cdot 2 \%$ of hemorrhagic strokes) had been admitted to hospital due to an IMD (Table 2). The risk of hemorrhagic stroke was significantly increased during the whole followup period for 15 of the 32 IMDs studied (Table 3). The overall risk of hemorrhagic stroke during the first year after hospitalization for an IMD was 2.65 (95 \% CI 2.27$3.08)$. The overall risk of hemorrhagic stroke decreased over time, to 1.83 after $1-5$ years (95\% CI 1.69-1.98), 1.63 after $5-10$ years (95\% CI 1.47-1.80) and 1.47 after $10+$ years (95\% CI 1.31-1.65).

The risk of hemorrhagic stroke was $\geq 2$ during the first year after hospitalization for seven IMD (Table 3): ankylosing spondylitis, immune thrombocytopenic purpura, polymyalgia rheumatica, psoriasis, rheumatoid arthritis, systemic lupus erythematosus, and Wegener's granulomatosis. For seven IMDs, the risk of hemorrhagic stroke was increased $10+$ years after hospitalization (Table 3): ankylosing spondylitis, celiac disease, Crohn's disease, Graves' disease, localized scleroderma, polymyalgia rheumatica, and rheumatoid arthritis.

\section{Hemorrhagic stroke and age and sex}

The overall risk of hemorrhagic stroke was increased for both sexes at all different follow-up periods (Additional file 1 Tables S2 and S3). The overall risk of hemorrhagic stroke was increased in all age groups for both males and females $(<50,50-59,60-69,70-79$, and $80+$ years $)$ (Additional file 1 Tables S4, S5 and S6).

\section{Ischemic stroke}

A total of 428,031 individuals were hospitalized with a main diagnosis of ischemic stroke (Table 2), of whom 9,437 ( $2 \cdot 2 \%$ of all ischemic stroke cases) had been admitted to hospital due to an IMD (Table 2). The variables for which the SIRs were adjusted are presented in Table 1. The risk of ischemic stroke was increased during the whole follow-up period for 20 of the 32 IMDs studied (Table 4). The overall risk of ischemic stroke during the first year after hospitalization for an IMD was $2 \cdot 02$ (95 \% CI 1.90-2.14). The overall risk of ischemic stroke decreased over time, to1.50 after $1-5$ years (95\% CI 1.46-1.55), 1.38 after 510 years (95\% CI $1.33-1.43$ ) and 1.29 after $10+$ years (95\% CI 1.23-1·35) (Table 4).

The risk of ischemic stroke was $\geq 2$ during the first year after hospitalization for twelve IMDs (Table 4): Addison's disease, Crohn's disease, Grave's disease, Hashimoto's thyroiditis, immune thrombocytopenic purpura, multiple sclerosis, polymyositis/dermatomyositis, rheumatic fever, rheumatoid arthritis, Sjögren's syndrome, systemic lupus erythematosus, and ulcerative colitis. For seven IMDs, the risk of ischemic stroke was increased $10+$ years after hospitalization: diabetes mellitus type 1, Graves' disease, Hashimoto's thyroiditis, pernicious anemia, polymyalgia rheumatica, psoriasis, and rheumatoid arthritis (Table 4).

\section{Ischemic stroke and age and sex}

The overall risk of ischemic or hemorrhagic stroke was increased for both sexes at all different follow-up periods (Additional file 1 Tables S7 and S8). The overall risk of ischemic stroke was increased in all age groups for both sexes (<50, 50-59, 60-69, 70-79, and 80+ years) (Additional file 1 Tables S9, S10 and S11).

\section{Time period and hemorrhagic and ischemic stroke}

The overall risk for both hemorrhagic and ischemic stroke was slightly higher between 1987 and 1996 (1.98 95 \% CI $1.78-2.20$ and $1.5195 \%$ CI 1.45-1.57, respectively) than between 1997 and 2008 (1.58 $95 \%$ CI 1.48-1.69 and 1.38 $95 \%$ CI 1.34-1.41, respectively) (Additional file 1 Tables S12 and S13).

\section{Discussion}

The present study is the first nationwide study of IMDs and ischemic and hemorrhagic stroke. The results indicate that several IMDs increase the risk of hospitalization for both ischemic and/or hemorrhagic stroke. The relative risk of ischemic and hemorrhagic stroke during the first year after hospitalization with certain IMDs was even higher than the risks associated with many traditional risk factors for ischemic and hemorrhagic stroke [1,25]. Although it declined over time, the overall risk of ischemic and hemorrhagic stroke remained elevated for 10 or more years for some IMDs. The results of our study are in line with previous studies linking rheumatoid arthritis [3,5,6,8-12,20], systemic lupus erythematosus [3,5,6,8,13-15,19], Sjögren's disease $[3,5,6,16]$, systemic vasculitis $[3,5]$, inflammatory bowel disease $[3,5,8,17]$, and psoriasis $[8,18]$ to an increased risk of 
Table 2 Number of cases of stroke, 1987-2008

\begin{tabular}{|c|c|c|c|c|c|c|c|}
\hline \multirow[b]{3}{*}{ Characteristics } & \multicolumn{4}{|c|}{ All stroke events } & \multicolumn{3}{|c|}{$\begin{array}{l}\text { Subsequent stroke } \\
\text { events of auto } \\
\text { immune disorders } \\
\text { patients }\end{array}$} \\
\hline & \multicolumn{3}{|c|}{$\begin{array}{l}\text { Hemorrhagic Ischeamic } \\
\text { stroke stroke }\end{array}$} & \multicolumn{4}{|c|}{$\begin{array}{l}\text { Hemorrhagic Ischeamic } \\
\text { stroke stroke }\end{array}$} \\
\hline & No. & $\%$ & No. $\quad \%$ & No. & $\%$ & No. & $\%$ \\
\hline \multicolumn{8}{|l|}{ Gender } \\
\hline Men & 36639 & 55.1 & 21680250.7 & 566 & 38.6 & 3216 & 34.1 \\
\hline Women & 29870 & 44.9 & 21122949.3 & 902 & 61.4 & 6221 & 65.9 \\
\hline \multicolumn{8}{|l|}{$\begin{array}{l}\text { Age at diagnosis } \\
\text { (yrs) }\end{array}$} \\
\hline$<50$ & 5070 & 7.6 & 136323.2 & 73 & 5.0 & 221 & 2.3 \\
\hline $50-59$ & 6857 & 10.3 & 292856.8 & 120 & 8.2 & 439 & 4.7 \\
\hline $60-69$ & 12399 & 18.6 & 7108916.6 & 249 & 17.0 & 1263 & 13.4 \\
\hline $70-79$ & 21079 & 31.7 & 14528833.9 & 476 & 32.4 & 3208 & 34.0 \\
\hline$>=80$ & 21104 & 31.7 & 16873739.4 & 550 & 37.5 & 4306 & 45.6 \\
\hline \multicolumn{8}{|l|}{$\begin{array}{l}\text { Period of diagnosis } \\
\text { (yrs) }\end{array}$} \\
\hline $1987-91$ & 13074 & 19.7 & $90088 \quad 21.0$ & 134 & 9.1 & 832 & 8.8 \\
\hline 1992-96 & 15114 & 22.7 & 11052525.8 & 299 & 20.4 & 2183 & 23.1 \\
\hline 1997-01 & 15773 & 23.7 & 9799322.9 & 389 & 26.5 & 2626 & 27.8 \\
\hline 2002-08 & 22548 & 33.9 & 12942530.2 & 646 & 44.0 & 3796 & 40.2 \\
\hline \multicolumn{8}{|l|}{$\begin{array}{l}\text { Socioeconomic } \\
\text { status }\end{array}$} \\
\hline Farmers & 4797 & 7.2 & 343758.0 & 109 & 7.4 & 773 & 8.2 \\
\hline Self-employed & 4911 & 7.4 & 314977.4 & 95 & 6.5 & 654 & 6.9 \\
\hline Professionals & 4726 & 7.1 & 255616.0 & 70 & 4.8 & 438 & 4.6 \\
\hline White collar workers & s18912 & 28.4 & 11932027.9 & 476 & 32.4 & 2773 & 29.4 \\
\hline Workers & 29487 & 44.3 & 19783346.2 & 653 & 44.5 & 4406 & 46.7 \\
\hline Others & 3676 & 5.5 & 194454.5 & 65 & 4.4 & 393 & 4.2 \\
\hline \multicolumn{8}{|l|}{ Region of residence } \\
\hline Big cities & 22409 & 33.7 & 14511933.9 & 429 & 29.2 & 2915 & 30.9 \\
\hline Northern Sweden & 12936 & 19.4 & 8068018.8 & 310 & 21.1 & 1972 & 20.9 \\
\hline Southern Sweden & 31164 & 46.9 & 20223247.2 & 729 & 49.7 & 4550 & 48.2 \\
\hline \multicolumn{8}{|l|}{$\begin{array}{l}\text { Hospitalization for } \\
\text { obesity }\end{array}$} \\
\hline Yes & 114 & 0.2 & $703 \quad 0.2$ & 2 & 0.1 & 30 & 0.3 \\
\hline No & 66395 & 99.8 & 42732899.8 & 1466 & 99.9 & 9407 & 99.7 \\
\hline \multicolumn{8}{|l|}{$\begin{array}{l}\text { Hospitalization for } \\
\text { alcoholism }\end{array}$} \\
\hline Yes & 2265 & 3.4 & $7448 \quad 1.7$ & 45 & 3.1 & 143 & 1.5 \\
\hline No & 64244 & 96.6 & 42058398.3 & 1423 & 96.9 & 9294 & 98.5 \\
\hline \multicolumn{8}{|l|}{$\begin{array}{l}\text { Hospitalization for } \\
\text { chronic lower } \\
\text { respiratory diseases }\end{array}$} \\
\hline Yes & 2067 & 3.1 & 182874.3 & 63 & 4.3 & 613 & 6.5 \\
\hline No & 64442 & 96.9 & 40974495.7 & 1405 & 95.7 & 8824 & 93.5 \\
\hline
\end{tabular}

Table 2 Number of cases of stroke, 1987-2008 (Continued)

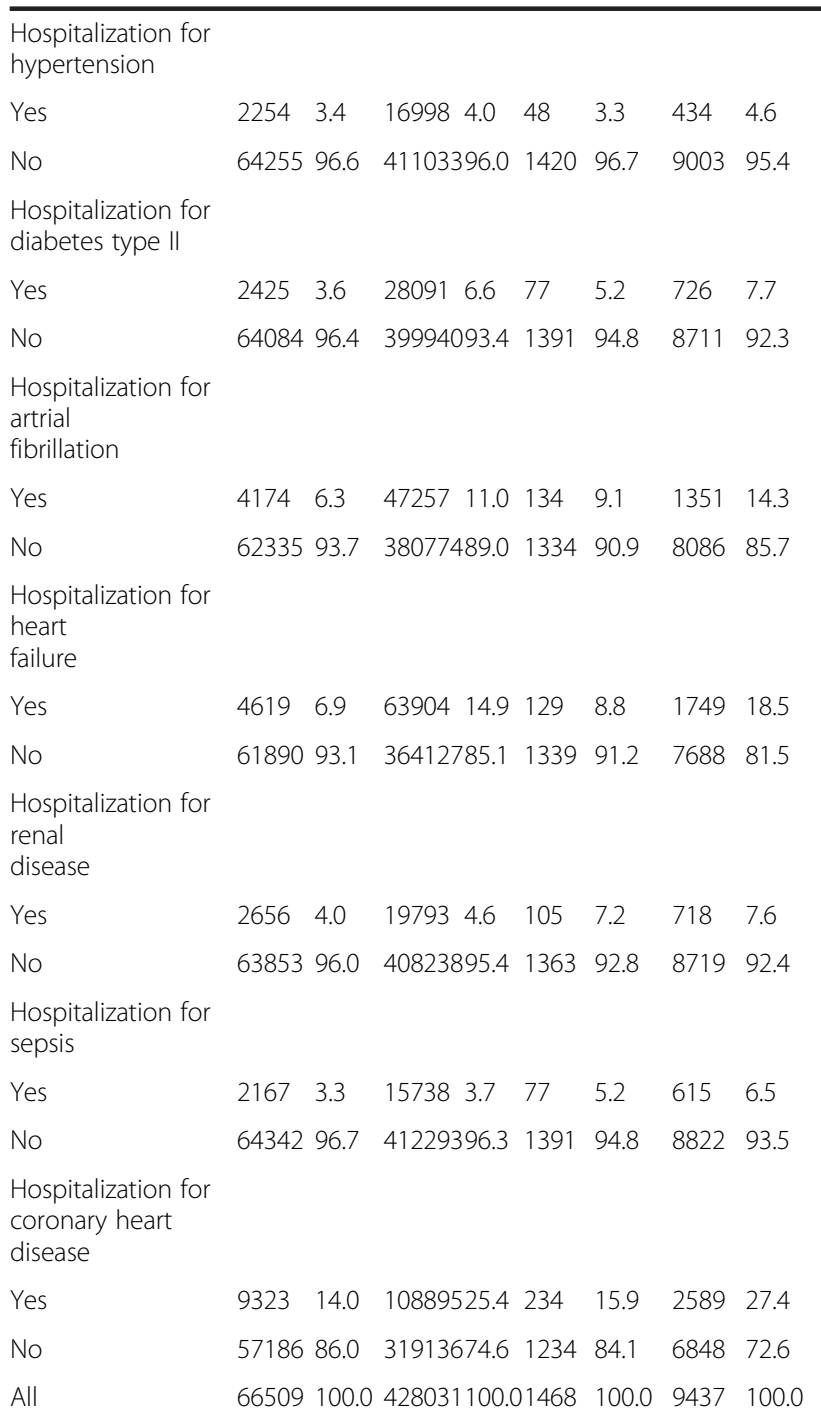

cardiovascular disease. However, what distinguishes our study from these other studies is its comparison of large numbers of patients and 32 types of IMDs with the general population in a nationwide setting, as well as the long-term follow-up of patients and the determination of risk for both ischemic and hemorrhagic stroke. Moreover, we also found a number of novel associations between IMDs and ischemic and hemorrhagic stroke. The results of the present study suggest that increased risk of subsequent ischemic and hemorrhagic stroke is a common feature of several IMDs, not just selected conditions such as systemic lupus erythematosus [19] and rheumatoid arthritis [20].

Although the increased risk of ischemic and hemorrhagic stroke may have different underlying causes in different IMDs, a general link between systemic inflammation and atherothrombosis has been indicated [2-8]. In some conditions, such as in immune thrombocytopenic purpura, 
Table 3 SIR for subsequent hemorrhagic stroke of patients with IMD

\begin{tabular}{|c|c|c|c|c|c|c|c|c|c|c|c|c|c|c|c|c|c|c|c|c|}
\hline \multirow{4}{*}{$\begin{array}{l}\text { Immune-mediated } \\
\text { diseases } \\
\text { Addison's disease }\end{array}$} & \multicolumn{20}{|c|}{ Follow-up interval (years) } \\
\hline & \multicolumn{4}{|l|}{$<1$} & \multicolumn{4}{|l|}{$1-5$} & \multicolumn{4}{|c|}{$5-10$} & \multicolumn{4}{|c|}{$>=10$} & \multicolumn{4}{|l|}{ All } \\
\hline & $\mathrm{O}$ & SIR & \multicolumn{2}{|c|}{$95 \% \mathrm{Cl}$} & $\mathrm{O}$ & SIR & \multicolumn{2}{|c|}{$95 \%$ Cl } & $\mathrm{O}$ & SIR & \multicolumn{2}{|c|}{$95 \% \mathrm{Cl}$} & $\mathrm{O}$ & SIR & \multicolumn{2}{|c|}{$95 \% \mathrm{Cl}$} & $\mathrm{O}$ & SIR & \multicolumn{2}{|c|}{$95 \% \mathrm{Cl}$} \\
\hline & 2 & 2.70 & 0.25 & 9.94 & 2 & 0.64 & 0.06 & 2.34 & 1 & 0.42 & 0.00 & 2.41 & 1 & 0.55 & 0.00 & 3.17 & 6 & 0.74 & 0.27 & 1.63 \\
\hline $\begin{array}{l}\text { Amyotrophic lateral } \\
\text { clerosis }\end{array}$ & 1 & 0.47 & 0.00 & 2.68 & 2 & 1.30 & 0.12 & 4.78 & 1 & 1.82 & 0.00 & 10.42 & 0 & & & & 4 & 0.89 & 0.23 & 2.30 \\
\hline nkylosin & 6 & 8.11 & 2.92 & 17.76 & 15 & 3.43 & 1.92 & 5.68 & 7 & 1.66 & 0.66 & 3.44 & 14 & 2.28 & 1.24 & 3.84 & 42 & 2.72 & 1.96 & 3.67 \\
\hline $\begin{array}{l}\text { Autoimmune } \\
\text { nemia }\end{array}$ & 1 & 3.13 & 0.00 & 17.91 & 4 & 2.96 & 0.77 & 7.66 & 1 & 1.15 & 0.00 & 6.59 & 2 & 2.99 & 0.28 & 10.98 & 8 & 2.49 & 1.06 & 4.93 \\
\hline Behcet's disease & 1 & 33.33 & 0.01 & 191.08 & 0 & & & & 0 & & & & 0 & & & & 1 & 1.67 & 0.00 & 9.55 \\
\hline eliac & 2 & 3.57 & 0.34 & 13.13 & 6 & 2.08 & 0.75 & 4.56 & 7 & 2.69 & 1.07 & 5.58 & 8 & 2.65 & 1.13 & 5.25 & 23 & 2.54 & 1.61 & 3.81 \\
\hline Chorea minor & 0 & & & & 0 & & & & 0 & & & & 0 & & & & 0 & & & \\
\hline rohn & 5 & 1.47 & 0.46 & 3.46 & 47 & 2.60 & 1.91 & 3.46 & 19 & 1.19 & 0.72 & 1.87 & 24 & 1.57 & 1.00 & 2.34 & 95 & 1.80 & 1.46 & 2.21 \\
\hline Diabetes mellitus type I & 0 & & & & 2 & 2.50 & 0.24 & 9.19 & 1 & 1.11 & 0.00 & 6.37 & 2 & 1.01 & 0.09 & 3.70 & 5 & 1.32 & 0.42 & 3.10 \\
\hline $\begin{array}{l}\text { Discoid } \\
\text { erythem }\end{array}$ & 1 & 11.11 & 0.00 & 63.69 & 1 & 2.33 & 0.00 & 13.33 & 0 & & & & 0 & & & & 2 & 1.87 & 0.18 & 6.87 \\
\hline e & 8 & 1.53 & 0.65 & 3.02 & 58 & 1.77 & 1.35 & 2.29 & 48 & 1.61 & 1.18 & 2.13 & 41 & 1.48 & 1.06 & 2.02 & 155 & 1.62 & 1.38 & 1.90 \\
\hline Hashimoto's thyro & 4 & 1.47 & 0.38 & 3.79 & 27 & 2.01 & 1.32 & 2.93 & 19 & 2.10 & 1.26 & 3.29 & 11 & 1.37 & 0.68 & 2.46 & 61 & 1.84 & 1.40 & 2.3 \\
\hline $\begin{array}{l}\text { nmune } \\
\text { hrombocytopenic } \\
\text { urpura }\end{array}$ & 8 & 8.60 & 3.67 & 17.03 & 12 & 2.81 & 1.45 & 4.92 & 6 & 2.18 & 0.79 & 4.78 & 2 & 1.23 & 0.12 & 4.54 & 28 & 2.93 & 1.94 & 4.2 \\
\hline Localized scleroderma & 0 & & & & 1 & 0.93 & 0.00 & 5 & 1 & 0.85 & 00 & 4.90 & 6 & 4.32 & 1.55 & 9.46 & 8 & 11 & 0.90 & 4.17 \\
\hline Lupoid hepatitis & 0 & & & & 0 & & & & 0 & & & & 0 & & & & 0 & & & \\
\hline lultiple sclerosis & 4 & 1.82 & 0.47 & 4.70 & 15 & 1.36 & 0.76 & 2.25 & 9 & 1.09 & 0.49 & 2.08 & 6 & 0.94 & 0.34 & 2.06 & 34 & 1.22 & 0.84 & 1.71 \\
\hline Myasthenia gravis & 1 & 1.22 & 0.00 & 6.99 & 8 & 2.09 & 0.89 & 4.14 & 6 & 2.17 & 0.78 & 4.76 & 0 & & & & 15 & 1.62 & 0.90 & 2.68 \\
\hline emia & 4 & 2.15 & 0.56 & 5.56 & 18 & 1.67 & 0.99 & 2.65 & 13 & 1.52 & 0.81 & 2.61 & 7 & 0.99 & 0.39 & 2.06 & 42 & 1.49 & 1.07 & 2.0 \\
\hline 1. & 2 & 5. & 0.51 & 19 & 0 & 0.00 & 0.54 & 2. & 3 & 1.91 & 0.36 & 5.66 & 0 & & & & 5 & 1.00 & 0.31 & 2.34 \\
\hline Polymyalgia rheumatica & 21 & 2.06 & 1.28 & 3.16 & 78 & 1.42 & 1.12 & 1.77 & 65 & 1.67 & 1.29 & 2.13 & 40 & 1.49 & 1.06 & 2.03 & 204 & 1.56 & 1.35 & 1.79 \\
\hline $\begin{array}{l}\text { Dlymy } \\
\text { ermat }\end{array}$ & 1 & 2.63 & 0.00 & 15.08 & 3 & 1.95 & 0.37 & 5.77 & 1 & 1.18 & 0.00 & 6.74 & 1 & 2.08 & 0.00 & 11.94 & 6 & 1.85 & 0.66 & 4.04 \\
\hline $\mathrm{im}$ & 1 & 2.08 & 0.00 & 1 & 3 & 1.76 & 0.33 & 5.22 & 2 & 2.17 & 0.20 & 7.99 & 0 & & & & 6 & 1.87 & 0.67 & 4.1 \\
\hline Psoriasis & 9 & 2.88 & 1.31 & 5.50 & 32 & 1.83 & 1.25 & 2.59 & 23 & 1.51 & 0.95 & 2.26 & 21 & 1.32 & 0.81 & 2.02 & 85 & 1.64 & 1.31 & 2.03 \\
\hline jite & 0 & & & & 1 & 2.94 & 0.00 & 16.86 & 1 & 2.56 & 0.00 & 14.70 & 0 & & & & 2 & 1.42 & 0.13 & 5.22 \\
\hline neum & 1 & 7.69 & 0.00 & 44.09 & 0 & & & & 0 & & & & 1 & 1.72 & 0.00 & 9.88 & 2 & 1.04 & 0.10 & 3.81 \\
\hline Rheumatoid arthritis & 65 & 3.27 & 2.52 & 4.17 & 191 & 2.03 & 1.76 & 2.34 & 109 & 1.92 & 1.57 & 2.31 & 61 & 1.78 & 1.36 & 2.29 & 426 & 2.08 & 1.89 & 2.29 \\
\hline $\operatorname{arco}$ & 3 & 2.48 & 0.47 & 7.34 & 12 & 1.87 & 0.96 & 3.28 & 14 & 2.26 & 1.23 & 3.80 & 6 & 0.79 & 0.28 & 1.73 & 35 & 1.64 & 1.14 & 2. \\
\hline Sjögren's syndrome & 0 & & & & 3 & 1.35 & 0.25 & 4.00 & 2 & 1.03 & 0.10 & 3.77 & 0 & & & & 5 & 0.81 & 0.26 & 1.90 \\
\hline $\begin{array}{l}\text { Systemic } \\
\text { erythema }\end{array}$ & 0 & 8.65 & 3.92 & 16.50 & 13 & 2.89 & 1.53 & 4.95 & 4 & 1.17 & 0.31 & 3.03 & 6 & 1.91 & 0.69 & 4.19 & 32 & 2.65 & 1.81 & 3.7 \\
\hline ystemic sclerosis & 2 & 3.17 & 0.30 & 11.67 & 5 & 2.67 & 0.84 & 6.29 & 3 & 2.73 & 0.51 & 8.07 & 2 & 3.45 & 0.33 & 12.68 & 12 & 2.87 & 1.48 & 5.0 \\
\hline Ulcerative colitis & 7 & 1.45 & 0.57 & 3.00 & 40 & 1.45 & 1.03 & 1.97 & 28 & 1.21 & 0.80 & 1.74 & 33 & 1.44 & 0.99 & 2.02 & 108 & 1.37 & 1.13 & 1.6 \\
\hline $\begin{array}{l}\text { Wegener's } \\
\text { granulomatosis }\end{array}$ & 6 & 83 & 2.10 & 12.76 & 3 & 0.90 & 0.17 & 2.67 & 2 & 1.08 & 0.10 & 3.95 & 0 & & & & 11 & 1.47 & 0.73 & 2.63 \\
\hline All & 175 & 2.65 & 2.27 & 3.08 & 602 & 1.83 & 1.69 & 1.98 & 396 & 1.63 & 1.47 & 1.80 & 295 & 1.47 & 1.31 & 1.65 & 1468 & 1.75 & 1.66 & 10. \\
\hline
\end{tabular}

$\mathrm{O}=$ observed number of cases; $\mathrm{SIR}=$ standardized incidence ratio; $\mathrm{Cl}=$ confidence interval. Bold type: $95 \% \mathrm{Cl}$ does not include 1.00 .

Adjusted for age, period, socioeconomic status, region of residence, hospitalization of chronic lower respiratory diseases, obesity, alcoholism, hypertension, diabetes, atrial fibrillation, heart failure, renal disease, sepsis, and coronary heart disease. 
Table 4 SIR for subsequent ischemic stroke of patients with IMD

\begin{tabular}{|c|c|c|c|c|c|c|c|c|c|c|c|c|c|c|c|c|c|c|c|c|}
\hline \multirow[b]{3}{*}{$\begin{array}{l}\text { Immune-mediated } \\
\text { diseases }\end{array}$} & \multicolumn{20}{|c|}{ Follow-up interval (years) } \\
\hline & \multicolumn{4}{|l|}{$<1$} & \multicolumn{4}{|l|}{$1-5$} & \multicolumn{4}{|l|}{$5-10$} & \multicolumn{4}{|c|}{$>=10$} & \multicolumn{4}{|l|}{ All } \\
\hline & $\mathrm{O}$ & $\mathrm{SIR}$ & 506 & & $\mathrm{O}$ & $\mathrm{SIR}$ & $95 \%$ & & $\mathrm{O}$ & SIR & $5 \%$ & & $\mathrm{O}$ & SIR & $5 \%$ & & $\mathrm{O}$ & SIR & $\%$ & $\mathrm{bCl}$ \\
\hline Addison's disease & 14 & 1 & 1.48 & 4.56 & 28 & 1.17 & 0.78 & 1.69 & 30 & 0 & 1.28 & 2.72 & 11 & 0.97 & 0.48 & 1.74 & 83 & .48 & 1.18 & 1.8 \\
\hline $\begin{array}{l}\text { myo } \\
\text { leros }\end{array}$ & 7 & 0.53 & 0.21 & 1.10 & 16 & 1.52 & 0.87 & 2.47 & 7 & 1.77 & 0.70 & 3.67 & 2 & 1.15 & 0.11 & 4.23 & 32 & 1.09 & 0.74 & 1.54 \\
\hline litis & 8 & 1.62 & 0.69 & 3.21 & 44 & 1.55 & 1.13 & 2.08 & 24 & 0.98 & 0.63 & 1.46 & 35 & 1.08 & 0.75 & 1.50 & 111 & 1.23 & 1.01 & 1. \\
\hline $\begin{array}{l}\text { utoin } \\
\text { nemia }\end{array}$ & 4 & 1.45 & 0.38 & 3.75 & 12 & 1.00 & 0.51 & 1.75 & 19 & 2.51 & 1.51 & 3.93 & 5 & 1.23 & 0.39 & 2.90 & 40 & 1.51 & 1.08 & 2.0 \\
\hline ehcet & 1 & 4.00 & 0.00 & 22.93 & 1 & 0.65 & 0.00 & 3.70 & 0 & & & & 1 & 1.43 & 0.00 & 8.19 & 3 & 0.78 & 0.15 & 2.29 \\
\hline 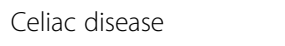 & 9 & 2.17 & 0.99 & 4.14 & 29 & 1.28 & 0.86 & 1.84 & 21 & 1.17 & 0.72 & 1.78 & 26 & 1.47 & 0.96 & 2.15 & 85 & 1.36 & 1.09 & 1.6 \\
\hline Chorea minor & 0 & & & & 1 & 2.27 & 0.00 & 13.03 & 0 & & & & 0 & & & & 1 & 1.11 & 0.00 & 6.37 \\
\hline Crohn disease & 49 & 2.15 & 1.59 & 2.84 & 160 & 1.33 & 1.13 & 1.55 & 103 & 1.11 & 0.91 & 1.35 & 97 & 1.15 & 0.93 & 1.40 & 409 & 1.28 & 1.16 & 1.4 \\
\hline iabete & 1 & 6.25 & 0.00 & 35.83 & 2 & 0.45 & 0.04 & 1.65 & 5 & 2.75 & 0.87 & 6.46 & 17 & 5.00 & 2.91 & 8.02 & 25 & 2.54 & 1.64 & 3.7 \\
\hline issoi & 3 & 4 & 0.80 & 12.51 & 3 & 0.99 & 0.19 & 2.92 & 1 & 0.47 & 0.00 & 2.70 & 3 & 1.55 & 0.29 & 4.60 & 10 & 1.28 & 0.61 & 2.3 \\
\hline ave & r & 2.15 & 1.76 & 2.62 & 402 & 1.39 & 1.26 & 1.53 & 348 & 1.36 & 1.22 & 1.51 & 276 & 1.27 & 1.12 & 1.43 & 1127 & 1.39 & 1.31 & 1. \\
\hline is & 77 & 2.99 & 2.36 & 3.74 & 211 & 1.73 & 1.50 & 1.98 & 115 & 1.39 & 1.14 & 1.67 & 82 & 1.28 & 1.02 & 1.59 & 485 & 1.64 & 1.50 & 1.8 \\
\hline $\begin{array}{l}\text { imune } \\
\text { irombocytopenic } \\
\text { urpura }\end{array}$ & 16 & ? & 1.34 & 3.83 & 55 & 1.77 & 1.33 & 2.30 & 19 & 0.94 & 0.57 & 1.48 & 14 & 1.20 & 0.65 & 2.01 & 104 & 1.49 & 1.22 & 1. \\
\hline 年 & 2 & 1.28 & 0.12 & 4.71 & 13 & 1.25 & 0.66 & 2 & 18 & 1.72 & 1.01 & 2.72 & 11 & 1.04 & 0.51 & 1.86 & 年 & .33 & 0.97 & 1.7 \\
\hline 15 & 3 & 4.48 & 0.84 & 13.25 & 4 & 1.98 & 0.52 & 5.12 & 0 & & & & 0 & & & & 7 & 2.10 & 0.83 & 4.36 \\
\hline ulti & 40 & 3.05 & 2.18 & 4.15 & 73 & 1.09 & 0.85 & 1.37 & 55 & 1.11 & 0.83 & 1.44 & 35 & 0.95 & 0.66 & 1.32 & 203 & 1.22 & 1.06 & 1.4 \\
\hline yes & 6 & 1.01 & 0.36 & 2.21 & 38 & 1.36 & 0.96 & or & 20 & 20 & 0.76 & 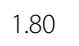 & 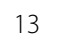 & 1.08 & ( & .85 & 00 & 1.23 & 0.97 & 1. \\
\hline nia & 25 & 1.56 & 1.01 & 2.31 & 138 & 1.49 & 1.25 & 1.76 & 89 & 1.23 & 0.99 & 1.52 & 74 & 1.44 & 1.13 & 1.80 & 326 & 1.40 & 1.25 & 1.5 \\
\hline (2) & J & 1.23 & 0.39 & 2.89 & 20 & 1.30 & 0.79 & 2. & 1 & 1.05 & 0.52 & 1.88 & 10 & 1.13 & 0.54 & 2.08 & $\Delta 6$ & 110 & 0.87 & 1. \\
\hline olym & 165 & 1.76 & 1.50 & 2.05 & 761 & 1.50 & 1.39 & 1.61 & 529 & 1.54 & 1.41 & 1.68 & 322 & 1.53 & 1.37 & 1.71 & 1777 & 1.54 & 1.47 & 1.6 \\
\hline $\begin{array}{l}\text { olymy } \\
\text { erma }\end{array}$ & 10 & 3.46 & 1.65 & 6.39 & 13 & 1.19 & 0.63 & 2.03 & 6 & 1.07 & 0.38 & 2.34 & 5 & 1.73 & 0.55 & 4.07 & 34 & 1.52 & 1.05 & 2.1 \\
\hline Primary biliary cirrhosis & 4 & 1.54 & 0 & 3.98 & 11 & 1.45 & 0.72 & 2 & 4 & 0.91 & 0.24 & 2.35 & 1 & 2.17 & 0.00 & 12.46 & 20 & 1 & 0.81 & 2. \\
\hline SOIIdSIS & 44 & 1.92 & 1.39 & 2.58 & 217 & 1.65 & 1.44 & 1.89 & 163 & 1.53 & 1.30 & 1.78 & 144 & 1.41 & 1.19 & 1.66 & 568 & 1.56 & 1.44 & 1.7 \\
\hline Reiter's dis & 0 & & & & 5 & 2.02 & 0.64 & 4.76 & 6 & 2.47 & 0.89 & 5.41 & 2 & 0.56 & 0.05 & 2.07 & 13 & 1.47 & 0.78 & 2.52 \\
\hline Rneumatic tever & 5 & 3.91 & 1 & 9.19 & 10 & 6 & 9 & 3 & 1 & 3.04 & 1.65 & 5.11 & 7 & 1.81 & 0.72 & 3.75 & 30 & 2.28 & 1.59 & 3. \\
\hline Rheur & 345 & 2.08 & 1.86 & 2.31 & 1266 & 1.66 & 1.57 & 1.75 & 663 & 1.45 & 1.34 & 1.56 & 326 & 1.30 & 1.16 & 1.45 & 2600 & 1.59 & 1.53 & 1.6 \\
\hline 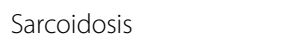 & 9 & 0.97 & 0.44 & 1.85 & 70 & 1.43 & 1.12 & 1.81 & 51 & 1.12 & 0.83 & 1.47 & 56 & 1.08 & 0.81 & 1.40 & 186 & 1.19 & 1.03 & 1. \\
\hline ögre & 10 & 2.57 & 1.22 & 4.75 & 28 & 1.38 & 0.92 & 1.99 & 15 & 0.96 & 0.54 & 1.59 & 15 & 1.26 & 0.70 & 2.08 & 68 & 1.31 & 1.02 & 1. \\
\hline us & 19 & 2.21 & 1.33 & 3.46 & 88 & 2.33 & 1.87 & 2.87 & 54 & 1.92 & 1.44 & 2.51 & 30 & 1.26 & 0.85 & 1.80 & 191 & 1.94 & 1.68 & 2.2 \\
\hline Systemic scierusis & 11 & 90 & 94 & 41 & 28 & 1.22 & 0.81 & 1 & 11 & 19 & 0.59 & 2.14 & 2 & 0.39 & 0.04 & 1.43 & 52 & 1.21 & 0.90 & 1.58 \\
\hline colitis & 71 & 2.15 & 1.68 & 2.71 & 231 & 1.27 & 1.11 & 1.45 & 162 & 1.09 & 0.92 & 1.27 & 146 & 1.05 & 0.89 & 1.24 & 610 & 1.21 & 1.12 & 1.3 \\
\hline Vegener's & 11 & 1.66 & 0.82 & 2.98 & 12 & 0.47 & 0.24 & 0.83 & 26 & 1.54 & 1.00 & 2.25 & 12 & 1.69 & 0.87 & 2.96 & 61 & 1.09 & 0.83 & 1.40 \\
\hline All & 1075 & 2.02 & 1.90 & 14 & 3990 & 1.50 & 1.46 & 1.55 & 2 & 1.38 & 1.33 & 1.43 & 1780 & 1 & 1.23 & 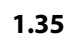 & 94 & & 1. & $1.42+3$ \\
\hline
\end{tabular}

$\mathrm{O}=$ observed number of cases; $\mathrm{SIR}=$ standardized incidence ratio; $\mathrm{Cl}=$ confidence interval.

Bold type: $95 \% \mathrm{Cl}$ does not include 1.00 .

Adjusted for age, period, socioeconomic status, region of residence, hospitalization of chronic lower respiratory diseases, obesity, alcoholism, hypertension, diabetes, atrial fibrillation, heart failure, renal disease, sepsis, and coronary heart disease. 
hemorrhagic stroke may occur as the direct result of thrombocytopenia. The formation of autoantibodies may, in special cases, also contribute to stroke [26]. The increased risk of stroke may be specific for more severe cases of IMDs, since the patients in our study had been admitted to hospital. The effects of treatment-corticosteroids promote hemostasis [27] — and the effect of inflammation on coagulation [7] may also contribute to the identified associations. Hypothetically, the fact that the risk of ischemic and hemorrhagic stroke decreased over time may suggest that it is linked to the inflammatory activity of the IMDs, which is likely to decrease over time due to treatment. In line with this hypothesis, in several studies disease activity appears to be linked with atherosclerosis progression $[2-8,28,29]$. However, as we lack treatment data, we cannot prove this hypothesis but in this context it is interesting that the relative risk of both hemorrhagic and ischemic stroke was lower between 1997 and 2008 than between 1987 and 1996 (Additional file 1 Tables S12 and S13).

The present study has certain limitations. For example, we had no data on general cardiovascular disease risk factors such as weight, smoking, and diet. It is unrealistic to gather such data for an entire national population. However, we did adjust for socioeconomic status, which is associated with risk factors such as smoking. Aspirin and nonsteroidal anti-inflammatory drugs (NSAID) may affect the risk of ischemic and hemorrhagic stroke [30,31]. However, we had no access to treatment data. Adjustment was, however, made for several comorbidities (chronic lower respiratory diseases, obesity, alcoholism and alcohol-related liver disease, type 2 diabetes mellitus, hypertension, atrial fibrillation, coronary heart diseases, heart failure, renal disease and sepsis). Still, residual bias may remain due to hospitalization of the most severe cases with IMD. However, all cases with previous or coexisting stroke were excluded to avoid selection bias. Totally, 8113 IMD patients with previous or coexisting ischemic stroke and 1416 with hemorrhagic stroke were excluded from the study, which in turn instead may underestimate the stroke risk. In fact, our results are within the limit for published cardiovascular disease risk in IMDs like RA [3,5,6,8-12,20] and SLE [3,5,6,8,13-15,19]. Thus, the estimated risks of stroke in IMD patients appear to be fairly valid. Anyway, the present study reflects the real world risks for stroke among hospitalized IMD (without previous stroke or at the same time as first hospitalization for IMD). All cases of ischemic and hemorrhagic stroke in Sweden should, according to official guidelines, be treated at hospitals [32]. Moreover, hospitalization incidence rates were calculated for the whole follow-up period, divided into five time periods, and adjustments were made for possible changes in hospitalization rates over time.

This study also has a number of strengths. The study reflects the situation in real world medicine during 22 years in a country with a high standard in the medical diagnosis [22,23,33-35]. The study population included all individuals clinically diagnosed with IMD and ischemic and hemorrhagic stroke in hospital during the study period, which eliminated recall bias. Because of the personal identification number assigned to each resident in Sweden, it was possible to trace all subjects for the whole follow-up period. Data on occupation were $99.2 \%$ complete (1980 and 1990 censuses), which enabled us to adjust our models for socioeconomic status. A further strength of the present study was the use of validated hospital discharge data. The Hospital Discharge Register has high validity [22,23,33-35], especially for cardiovascular disorders such as stroke, for which approximately $95 \%$ of diagnoses have been shown to be correct [3335]. Though, the positive predictive value (PPV) may differ between diagnoses in the Swedish Hospital Discharge Register, the PPV is generally around 85-95 \% [35].

\section{Conclusions}

In summary, the risk of hospitalization for ischemic and hemorrhagic stroke was, for several immune-mediated diseases studied, found to be significantly associated. The risk of ischemic and hemorrhagic stroke during the first year after hospitalization with an immune-mediated disease was high for certain IMDs. Although it decreased over time, for some IMDs the risk of ischemic and hemorrhagic stroke remained elevated for more than 10 years. The findings of the present study suggest that many IMDs are linked to cerebrovascular disease. Future studies could elucidate the mechanisms behind stroke in specific IMDs.

\section{Additional file}

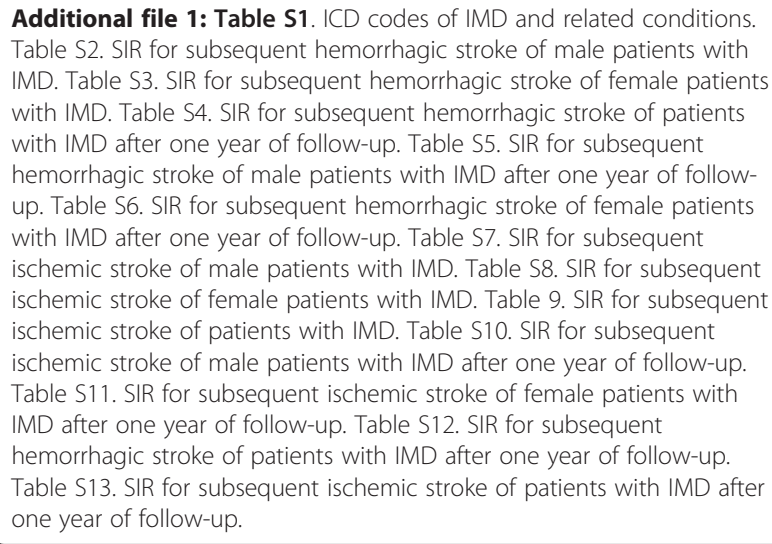

Additional file 1: Table S1. ICD codes of IMD and related conditions. Table S2. SIR for subsequent hemorrhagic stroke of male patients with IMD. Table S3. SIR for subsequent hemorrhagic stroke of female patients with IMD. Table S4. SIR for subsequent hemorrhagic stroke of patients with IMD after one year of follow-up. Table S5. SIR for subsequent hemorrhagic stroke of male patients with IMD after one year of followup. Table S6. SIR for subsequent hemorrhagic stroke of female patients with IMD after one year of follow-up. Table S7. SIR for subsequent ischemic stroke of male patients with IMD. Table S8. SIR for subsequent ischemic stroke of female patients with IMD. Table 9. SIR for subsequent ischemic stroke of patients with IMD. Table S10. SIR for subsequent ischemic stroke of male patients with IMD after one year of follow-up. Table S11. SIR for subsequent ischemic stroke of female patients with IMD after one year of follow-up. Table S12. SIR for subsequent hemorrhagic stroke of patients with IMD after one year of follow-up. Table S13. SIR for subsequent ischemic stroke of patients with IMD after one year of follow-up.

\section{Abbreviations}

$\mathrm{Cl}$ : Confidence interval; E: Expected; ICD: international classification of diseases; IMD: immune-mediated disease; O: Observed; RA: rheumatoid arthritis; SES: socioeconomic status; SIR: standarized incidence ratio; SLE: systemic lupus erythematosus.

\section{Competing interests}

The authors declare that they have no competing interests. 


\section{Authors' contributions}

All authors contributed to the conception and design of the study; JS and KS contributed to the acquisition of data; all authors contributed to the analysis and interpretation of data; BZ drafted the manuscript; and all authors revised it critically and approved the final version. All authors had full access to all of the data (including statistical reports and tables) and take responsibility for the integrity of the data and the accuracy of its analysis.

\section{Acknowledgements}

The authors wish to thank the CPF's Science Editor Stephen Gilliver for his useful comments on the text. The registers used in the present study are maintained by Statistics Sweden and the National Board of Health and Welfare. This work was supported by grants to Bengt Zöller from the Swedish Heart and Lung Foundation and Region Skåne (REGSKANE-124611), and to Kristina and Jan Sundquist from the Swedish Research Council (2008-3110 and 2008-2638), the Swedish Council for Working Life and Social Research (2006-0386, 2007-1754 and 2007-1962), and Formas (2006-4255-6596-99 and 2007-1352). No funding bodies played any role in the design, in the collection, analysis, and interpretation of data or in the writing and decision to publish this manuscript.

\section{Author details}

${ }^{1}$ Center for Primary Health Care Research, Lund University/Region Skåne, Clinical Research Centre, Floor 11, Building 28, Entrance 72, Skåne University Hospital, 205 02, Malmö, Sweden. ${ }^{2}$ Stanford Prevention Research Centre, Stanford University School of Medicine, Medical School Office Building, 251 Campus Drive, Mail Code 5411Stanford, California, 94305-5411, USA.

Received: 22 November 2011 Accepted: 18 June 2012

Published: 18 June 2012

\section{References}

1. Donnan GA, Fisher M, Macleod M, Davis SM: Stroke. Lancet 2008, 371:1612-23.

2. Libby P: Inflammation in atherosclerosis. Nature 2002, 420:868-74.

3. van Leuven SI, Franssen R, Kastelein JJ, Levi M, Stroes ES, Tak PP: Systemic inflammation as a risk factor for atherothrombosis. Rheumatology 2008, 47:3-7.

4. Hansson GK, Hermansson A: T. The immune system in atherosclerosis. Nature Immunology 2011, 12:204-212.

5. Shoenfeld Y, Gerli R, Doria A, Matsuura E, Cerinic MM, Ronda N, Jara LJ, Abu-Shakra M, Meroni PL, Sherer Y: Accelerated atherosclerosis in autoimmune rheumatic diseases. Circulation 2005, 112:3337-47.

6. López-Pedrera C, Pérez-Sánchez C, Ramos-Casals M, Santos-Gonzalez M, Rodriguez-Ariza A, José Cuadrado M: Cardiovascular risk in systemic autoimmune diseases. Clin Dev Immunol: epigenetic mechanisms of immune regulatory functions; 2012. In press.

7. $\mathrm{Xu} \mathrm{J}$, Lupu F, Esmon CT: Inflammation, innate immunity and blood coagulation. Hamostaseologie 2010, 30(5-6):8-9.

8. El-Gabalawy H, Guenther LC, Bernstein CN: Epidemiology of immunemediated inflammatory diseases: incidence, prevalence, natural history, and comorbidities. J Rheumatol Supp/ 2010, 85:2-10.

9. Lévy L, Fautrel B, Barnetche T: Schaeverbeke T. Incidence and risk of fatal myocardial infarction and stroke events in rheumatoid arthritis patients. A systematic review of the literature. Clin Exp Rheumatol 2008, 26:673-9.

10. Solomon DH, Karlson EW, Rimm EB, et al: Cardiovascular morbidity and mortality in women diagnosed with rheumatoid arthritis. Circulation 2003, 107:1303-7.

11. Gabriel SE: Cardiovascular morbidity and mortality in rheumatoid arthritis. Am J Med 2008, 121:S9-14.

12. Libby P: Role of inflammation in atherosclerosis associated with rheumatoid arthritis. Am J Med 2008, 121:S21-S31.

13. Manzi S, Meilahn EN, Rairie JE, et al: Age-specific incidence rates of myocardial infarction and angina in women with systemic lupus erythematosus: comparison with the Framingham Study. Am J Epidemiol 1997, 145:408-15.

14. Asanuma $Y$, Oeser A, Shintani AK, Turner E, Olsen N, Fazio S, Linton MF, Raggi $P$, Stein CM: Premature coronary-artery atherosclerosis in systemic lupus erythematosus. N Engl J Med 2003, 349:2407-15.

15. Roman MJ, Shanker BA, Davis A, et al: Prevalence and correlates of accelerated atherosclerosis in systemic lupus erythematosus. $N$ Engl Med 2003, 349:2399-406.
16. Vaudo G, Bocci EB, Shoenfeld $Y$, et al: Precocious intima-media thickening in patients with primary Sjogren's syndrome. Arthritis Rheum 2005, 52:3890-7.

17. van Leuven SI, Hezemans R, Levels JH, Snoek S, Stokkers PC, Hovingh GK, Kastelein JJ, Stroes ES, de Groot E, Hommes DW: Enhanced atherogenesis and altered high density lipoprotein in patients with Crohn's disease. $J$ Lipid Res 2007, 48:2640-6.

18. Vizzardi E, Raddino R, Teli M, Gorga E, Brambilla G: Dei Cas L. Psoriasis and cardiovascular diseases. Acta Cardiol. 2010, 65:337-40.

19. Krishnan E: Stroke subtypes among young patients with systematic lupus erythematosus. Am J Medicine 2005, 118:1415.

20. Nadareishvili Z, Michaud K, Hallenbeck JM, Wolfe F: Cardiovascular, rheumatologic, and pharmacologic predictors of stroke in patients with rheumatoid arthritis: a nested, case-control study. Arthritis Rheum 2008, 59:1090-6.

21. Zöller B, Li X, Sundquist J, Sundquist K: Age- and gender-specific familial risks for venous thromboembolism: a nationwide epidemiological study based on hospitalizations in Sweden. Circulation 2011, 124:1012-20.

22. Rosen M, Hakulinen T: Use of disease registers. In Handbook of epidemiology. Edited by Ahrens W, Pigeot I. Berlin: Springer; 2005:231-52.

23. The Swedish Hospital Discharge Register 1987-1996: quality and contents. Stockholm: The National Board of Health and Welfare;

24. Rothman KJ, Greenland S: Modern Epidemiology. 2nd edition. Philadelphia: Lippincott-Raven; 1998

25. O'Keefe $\mathrm{JH}$, Carter MD, Lavie CJ: Primary and secondary prevention of cardiovascular diseases: a practical evidence-based approach. Mayo Clin Proc 2009, 84:741-57.

26. Saidi S, Mahjoub T, Almawi WY: Lupus anticoagulants and antiphospholipid antibodies as risk factors for a first episode of ischemic stroke. J Thromb Haemost 2009, 7:1075-1080.

27. Jilma B, Cvitko T, Winter-Fabry A, Petroczi K, Quehenberger P, Blann AD: High dose dexamethasone increases circulating P-selectin and von Willebrand factor levels in healthy men. Thromb Haemost 2005, 94:797-801

28. Westlake SL, Colebatch AN, Baird J, et al: The effect of methotrexate on cardiovascular disease in patients with rheumatoid arthritis: a systematic literature review. Rheumatology 2010, 49:295-307.

29. Westlake SL, Colebatch AN, Baird J, Edwards CJ, et al: Tumour necrosis factor antagonists and the risk of cardiovascular disease in patients with rheumatoid arthritis: a systematic literature review. Rheumatology 2011, 50:518-31.

30. Roumie CL, Mitchel EF Jr, Kaltenbach L, Arbogast PG, Gideon P, Griffin MR: Nonaspirin NSAIDs, cyclooxygenase 2 inhibitors, and the risk for stroke. Stroke 2008, 39:2037-45.

31. He J, Whelton PK, Vu B, Klag MJ: Aspirin and risk of hemorrhagic stroke: a meta-analysis of randomized controlled trials. JAMA 1998, 280:1930-5.

32. National guidelines for stroke care 2009: Basis for control and management In Swedish: The National Board of Health and Welfare, Stockholm; 2009.

33. Validity of the diagnoses from the Swedish In-Care Register 1987 and 1995: National Board of Health and Welfare. In Swedish: Stockholm; 2000.

34. Lindblad U, Råstam L, Ranstam J, Peterson M: Validity of register data on acute myocardial infarction and acute stroke: the Skaraborg Hypertension Project. Scand J Soc Med 1993, 21:3-9.

35. Ludvigsson JF, Andersson E, Ekbom A, Feychting M, Kim JL, Reuterwall C, Heurgren M, Olausson PO: External review and validation of the Swedish national inpatient register. BMC Public Health 2011, 11:450.

doi:10.1186/1471-2377-12-41

Cite this article as: Zöller et al:: Risk of subsequent ischemic and hemorrhagic stroke in patients hospitalized for immune-mediated diseases: a nationwide follow-up study from Sweden. BMC Neurology 2012 12:41. 